\title{
Iron stores in relation to dietary patterns in a multiethnic population: the SAMINOR study
}

\author{
Ann R Broderstad*, Marita Melhus, Magritt Brustad and Eiliv Lund \\ Centre for Sami Health Research, Institute of Community Medicine, Faculty of Health Sciences, \\ University of Tromsø, N-9037 Tromsø, Norway
}

Submitted 21 September 2009: Accepted 20 October 2010: First published online 4 January 2011

\begin{abstract}
Objective: We evaluated the association between serum ferritin (s-ferritin), transferrin saturation and dietary patterns, in connection with ethnicity, geographical settlement and lifestyle factors.

Design: In 2003-2004, a cross-sectional study of health and living conditions was carried out in northern Norway.

Setting: A questionnaire explored, among other factors, ethnicity and food consumption habits. Principal component analysis was used to assess the association between variables. Seven principal components were then used as input to a cluster analysis. To characterise food consumptions, five dietary patterns were identified and used to assess the effect of food consumption habits on Fe stores. Subjects: A total of 16323 men and women between the ages of 36 and 79 years participated.

Results: Participants who frequently consumed reindeer meat had higher levels of s-ferritin $(P<0$.0001) than did individuals with other dietary patterns. This pattern was highly represented by subjects with three generations of Sami language (Sami I). Further, mean transferrin saturation in the reindeer group was higher compared with the other dietary clusters for men $(P<0 \cdot 04)$ and women $(P<0 \cdot 02)$. However, the reindeer pattern individuals also had the highest proportion of subjects with overweight and obesity. Obesity was positively associated with s-ferritin in both men and women $(P<0 \cdot 0001)$.

Conclusions: The differences in Fe status described earlier between inland Sami and non-Sami can be explained by several factors such as food habits, age and obesity. High level of s-ferritin may reflect high intake of reindeer meat. Being overweight and obese is also associated with s-ferritin levels.
\end{abstract}

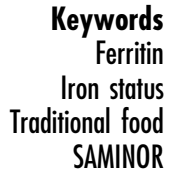

Ferritin SAMnion SAMINOR
The Norwegian government has formally designated the Sami people as the indigenous people of Norway. The Sami live in the northern part of Scandinavia and in the Kola Peninsula of Russia. Norway has the greatest proportion of the total Sami population. Previous studies have reported differences in Fe status between indigenous groups and the majority population, mainly because of differences in dietary Fe intake ${ }^{(1)}$. A study from areas with both Sami and non-Sami inhabitants in central and northern Norway described differences in Fe levels between Sami and non-Sami populations ${ }^{(2)}$. The inland Sami had significantly higher serum ferritin (s-ferritin) than the coastal Sami and non-Sami populations, independent of sex. In addition, mean transferrin saturation was higher for inland residents, especially for male participants.

In northern Norway, there has been substantial social interaction between the Sami and non-Sami populations for several generations. However, some special characteristics in dietary traditions have influenced the Sami dietary habits, such as high consumption of reindeer meat by inland Sami and marine fish by coastal dwellers.

The s-ferritin level is the most specific biochemical test that correlates with total body Fe stores, and has been used as a key parameter in several epidemiological studies to assess Fe status ${ }^{(2-5)}$. Serum apoferritin (s-apoferritin) is included in measured s-ferritin and is an acute-phase reactant protein elevated in infectious, inflammatory and malignant diseases. However, the recent SAMINOR study $^{(2)}$ on Fe levels indicated that acute infection or inflammation was not prevalent in the study population. The high mean Fe levels among inland Sami groups could not be explained by differences in alcohol consumption, since the Sami participants consumed less alcohol compared with non-Sami participants. A likely explanation is differences in dietary habits. 
The aim of the present study was to investigate whether dietary habits could account for the observed differences in Fe status between the Sami and other Norwegian participants in the SAMINOR survey.

\section{Methods}

\section{The SAMINOR study}

A cross-sectional population-based study of health and living conditions in areas with a mixed Sami and nonSami population, referred to as the SAMINOR study, was carried out in central and northern Norway in 2003-2004. The present study covered municipalities in Norway for which $>5-10 \%$ of the population was reported in the 1970 Census to be Sami ${ }^{(6)}$. In addition, some specific districts were included from municipalities with an overall lower proportion of subjects with Sami ethnicity. The SAMINOR study was the responsibility of the Centre for Sami Health Research, Institute of Community Medicine at the University of Troms $\varnothing$, in collaboration with the National Screening Program for Cardiovascular Diseases (SHUS; now incorporated into the National Institute of Public Health). The study is described in detail by Lund et $a l .{ }^{(7)}$. Study materials were collected from January 2003 to April 2004.

\section{Subjects}

In total, 16538 men and women aged 36-79 years participated in the SAMINOR study and gave informed consent for medical research. The response rate was $61 \%$. Participation rates for the coastal and inland areas were $59 \cdot 6 \%$ and $65.5 \%$, respectively. More women than men participated, $65 \cdot 6 \% v$. $56 \cdot 6 \%$, respectively. Information about ethnicity and Fe status was available for 14630 persons.

\section{Screening}

Non-fasting blood samples were obtained at enrolment. Blood samples were drawn by venepuncture at normal venous pressure in sitting position. Serum was separated immediately and s-ferritin, s-Fe and transferrin were measured by standard techniques used routinely in the clinical laboratory. s-Ferritin measurements were taken with a Hitachi Modular P analyser (Roche Diagnostics, Mannheim, Germany); details of the analytical methods used have been described previously by Broderstad et $a l^{(2)}$. In the current statistical analyses, we chose only $\mathrm{s}$-ferritin as a measure of Fe status so as to limit the length of the paper. Fe depletion is defined as s-ferritin concentrations $<13 \mu \mathrm{g} / \mathrm{l}$, which is consistent with earlier surveys $^{(3,8)}$. The WHO defines Fe depletion to occur when s-ferritin $<15 \mu \mathrm{g} / \mathrm{l}^{(9)}$.

Body weight (in $\mathrm{kg}$, one decimal) and height (in $\mathrm{cm}$, one decimal) were measured using an electronic height and weight scale with the participants wearing light clothing without shoes. According to WHO, underweight is defined as $\mathrm{BMI}<18.5 \mathrm{~kg} / \mathrm{m}^{2}$, standard weight as between 18.5 and $24.9 \mathrm{~kg} / \mathrm{m}^{2}$, overweight as between 25.0 and $29 \cdot 9 \mathrm{~kg} / \mathrm{m}^{2}$ and obese as $\geqslant 30.0 \mathrm{~kg} / \mathrm{m}^{2(10)}$.

C-reactive protein (CRP) concentrations were measured with a turbidimetric assay. The method was standardised against 470 (RPPHS - Reference Preparation for Proteins in Human Serum).

\section{FFQ data}

The dietary cluster analysis was based on FFQ data ${ }^{(11)}$. Respondents were asked to report their usual intake of the following foods: fruit, berries, cheese (all types), potatoes, cooked vegetables and raw vegetables/salad. Questions were asked about the usual intake of different beverages. In addition, the respondents were asked questions about traditional and modern food items assumed to be a part of the current diet of the study population.

\section{Dietary babits}

Information on consumption of different foods, both modern and traditional, was obtained through questionnaires with a food frequency design, as described in detail by Brustad et $a l^{(11)}$. On the basis of factor and cluster analyses for fifty-six different food variables, Brustad et al. ${ }^{(11)}$ defined five different dietary groupings or patterns: 'reindeer', 'fish', 'average', 'fruit and vegetables' and 'Westernised, traditional marine'. The reindeer pattern was characterised by frequent consumption of reindeer meat and other reindeer products, in addition to moose meat and cured/salted fish. The use of boiled (non-filtered) coffee was also frequent in this cluster. The second pattern was named 'fish' because it consisted of subjects with frequent use of all marine food items in the questionnaire. Pattern three was labelled 'average' and characterised by average intakes of most food items, except whole milk, and processed fish (smoked or cured/ salted), which were significantly higher in this group. In addition, this pattern showed a high intake of both boiled (non-filtered) and 'other' coffee, as well as sausages, pork and mutton. The fourth pattern was labelled 'fruit and vegetables' because of the frequent intake of these items in addition to water, tea, pasta and chicken. The last pattern was named 'Western, traditional marine'. This was dominated by Westernised products such as hamburgers, pizza, sausages, casseroles, pork and beef. This pattern also had the highest frequency of the traditional food, namely, fish liver and hard roe, in addition to whale meat and filtered coffee. These dietary patterns are used to assess the effect of food habits on Fe stores.

\section{Etbnicity}

The sample was divided into three ethnic groups: (i) Sami I - speaking the Sami language for three generations (all grandparents, both parents and the participants used Sami as the language spoken at home); (ii) Sami II mixed group with Sami and non-Sami affiliation (at least 
one Sami indicator as Sami language, ethnic background or self-perceived ethnicity); and (iii) the remaining population - non-Sami. Each of these groups was divided into inland and coastal domiciles.

\section{Reference groups}

The reference groups in the multiple linear regression analyses were the youngest age groups, the non-Sami group, those consuming the average dietary pattern and the normal weight group in each category.

\section{Ethical approval}

All participants gave written informed consent before screening. The study was accredited by the Regional Board of Research Ethics in Northern Norway, including the Sami consultant of the Board. The survey is in accordance with the Helsinki Declaration of 1975. The National Data Protection Authority (Datatilsynet) gave approval for storing of individual information and for later linkages.

\section{Statistical analyses}

Analyses were stratified by sex. Since distributions for s-ferritin showed positive skewness, concentrations were logarithmically transformed $(\log (e)$ scale), which resulted in normal distributions. s-Ferritin concentrations are reported as geometric means and their 95\% CI. Differences in Fe levels across ethnic groups were tested by ANOVA, stratified for geographical residence and two age groups (36-49 and 50-79 years). The Tukey-Kramer test was used for pairwise comparisons. ANCOVA was used to test for differences in Fe levels between geographical residences, adjusted for age group and ethnicity. Ageadjusted effects of ethnicity, BMI and dietary habits on log-ferritin were modelled by multiple linear regressions for inland residents only. Finally, log-ferritin was the dependent variable in a predefined multiple regression model, mutually adjusting for ethnicity, age group, BMI and dietary habits. We used the SAS statistical software package version 9.1 (SAS Institute, Cary, NC, USA).

\section{Results}

Table 1 shows selected characteristics of the study participants. Sami affiliation was reported by 4932 participants (34\%) for whom s-ferritin was available. Almost $80 \%$ of the individuals had BMI $>24.9 \mathrm{~kg} / \mathrm{m}^{2}$. Obesity rates were somewhat higher in women than in men: $21.5 \% v$. $17.4 \%$, respectively. Mean s-ferritin levels for men and women were 99 (95\% CI 97, 101) $\mu \mathrm{g} / 1$ and 42 (95\% CI 41, 43) $\mu \mathrm{g} / \mathrm{l}$, respectively.

Twenty-one $(0 \cdot 30 \%)$ men and twenty-two (0.29\%) women had CRP between 50 and $100 \mathrm{mg} / \mathrm{l}$. In addition, seven $(0.09 \%)$ men and five $(0.06 \%)$ women had $\mathrm{CRP}>100 \mathrm{mg} / \mathrm{l}$. None of them had s-ferritin $>300 \mu \mathrm{g} / \mathrm{l}$. The linear association between CRP and s-ferritin was weak but
Table 1 Selected characteristics of the study sample ( $n$ 14630)*

\begin{tabular}{|c|c|c|c|c|}
\hline \multirow[b]{2}{*}{ Characteristic } & \multicolumn{2}{|c|}{ Women } & \multicolumn{2}{|c|}{ Men } \\
\hline & $n$ & $\%$ & $n$ & $\%$ \\
\hline \multicolumn{5}{|l|}{ Age group (years) } \\
\hline $36-39$ & 765 & $10 \cdot 1$ & 606 & $8 \cdot 6$ \\
\hline $40-49$ & 2068 & $27 \cdot 4$ & 1843 & $26 \cdot 1$ \\
\hline $50-59$ & 2270 & $30 \cdot 0$ & 2253 & $31 \cdot 8$ \\
\hline $60-69$ & 1539 & $20 \cdot 4$ & 1524 & $21 \cdot 5$ \\
\hline $70-79$ & 916 & $12 \cdot 1$ & 846 & $12 \cdot 0$ \\
\hline \multicolumn{5}{|l|}{ Ethnicity } \\
\hline Sami I & 845 & $11 \cdot 2$ & 817 & $11 \cdot 5$ \\
\hline Sami II & 1623 & $21 \cdot 5$ & 1647 & $23 \cdot 3$ \\
\hline Non-Sami & 5090 & $67 \cdot 3$ & 4608 & $65 \cdot 2$ \\
\hline \multicolumn{5}{|l|}{ BMI $\left(\mathrm{kg} / \mathrm{m}^{2}\right)$} \\
\hline$<18.5$ & 40 & 0.5 & 16 & $0 \cdot 2$ \\
\hline $18 \cdot 5-24 \cdot 9$ & 1851 & $24 \cdot 5$ & 1121 & $15 \cdot 9$ \\
\hline $25 \cdot 0-29 \cdot 9$ & 4030 & $53 \cdot 5$ & 4697 & $66 \cdot 5$ \\
\hline$\geq 30 \cdot 0$ & 1618 & $21 \cdot 5$ & 1228 & $17 \cdot 4$ \\
\hline \multicolumn{5}{|l|}{ Dietary pattern } \\
\hline Reindeer & 370 & $5 \cdot 8$ & 376 & $6 \cdot 4$ \\
\hline Fish & 980 & $15 \cdot 2$ & 705 & $11 \cdot 9$ \\
\hline Average & 1404 & $21 \cdot 9$ & 1710 & $28 \cdot 9$ \\
\hline Fruit/vegetables & 1667 & $26 \cdot 0$ & 700 & $11 \cdot 8$ \\
\hline Western/marine & 1999 & $31 \cdot 1$ & 2422 & $41 \cdot 0$ \\
\hline \multicolumn{5}{|c|}{ Ethnicity by geographical area } \\
\hline Sami (inland) & 734 & $9 \cdot 7$ & 669 & $9 \cdot 4$ \\
\hline Sami (coastal) & 1734 & $22 \cdot 9$ & 1795 & $24 \cdot 4$ \\
\hline Norwegian (inland) & 769 & $10 \cdot 2$ & 678 & $9 \cdot 6$ \\
\hline Norwegian (coastal) & 4321 & $57 \cdot 2$ & 3930 & $55 \cdot 6$ \\
\hline
\end{tabular}

*Subgroups may not add up to 14630 for all variables because of missing values.

significant $(r=0 \cdot 06, P<0 \cdot 0001)$. Seven individuals had sferritin $>1000 \mu \mathrm{g} / \mathrm{l}$, but all had CRP $<10 \mathrm{mg} / \mathrm{l}$.

\section{Distribution of Fe stores}

The mean s-ferritin level was significantly higher for men than for women, independent of ethnic affiliation. Levels by age group, ethnic and geographical categories (both sex) are presented in Table 2. Regardless of ethnicity, s-ferritin levels for men declined with advancing age, whereas they increased after the age of 50 years for women. There was a significantly higher s-ferritin concentration among inland participants compared with coastal residents (both sexes). For inland men, the Sami I group aged 36-49 years had a significantly higher mean s-ferritin than the Sami II group (Tukey-Kramer test). The Sami I group also had significantly higher mean s-ferritin than the non-Sami group (Tukey-Kramer test). Inland women older than 50 years in the Sami I group had significantly higher mean s-ferritin than the corresponding Sami II and non-Sami groups (Tukey-Kramer test). Table 3 shows mean s-ferritin by dietary patterns. The reindeer group had significant higher mean s-ferritin in both sexes when adjusted for age.

For the coastal settlements, there were no differences in mean s-ferritin between ethnic categories. Table 4 presents mean transferrin saturation by dietary patterns. Transferrin saturation showed less variation than s-ferritin both among men and women. Mean transferrin saturation in the reindeer group was slightly higher compared with 
Table 2 s-Ferritin by geographical area and ethnicity for 14630 participants in northern Norway: the SAMINOR survey

\begin{tabular}{|c|c|c|c|c|c|c|c|c|c|c|c|c|c|}
\hline & \multicolumn{13}{|c|}{$\mathrm{s}$-Ferritin $(\mu \mathrm{g} / \mathrm{l})$} \\
\hline & \multicolumn{6}{|c|}{ Inland } & \multicolumn{6}{|c|}{ Coastal } & \multirow[b]{3}{*}{$P$ value* } \\
\hline & \multicolumn{2}{|c|}{ Sami I } & \multicolumn{2}{|c|}{ Sami II } & \multicolumn{2}{|c|}{ Non-Sami } & \multicolumn{2}{|c|}{ Sami I } & \multicolumn{2}{|c|}{ Sami II } & \multicolumn{2}{|c|}{ Non-Sami } & \\
\hline & Mean & $95 \% \mathrm{Cl}$ & Mean & $95 \% \mathrm{Cl}$ & Mean & $95 \% \mathrm{Cl}$ & Mean & $95 \% \mathrm{Cl}$ & Mean & $95 \% \mathrm{Cl}$ & Mean & $95 \% \mathrm{Cl}$ & \\
\hline Men & \multicolumn{2}{|c|}{ (n 452) } & \multicolumn{2}{|c|}{$(n 217)$} & \multicolumn{2}{|c|}{$(n 690)$} & \multicolumn{2}{|c|}{$(n 365)$} & \multicolumn{2}{|c|}{$(n$ 1430) } & \multicolumn{2}{|c|}{ (n 4001) } & \\
\hline $\begin{array}{l}\text { Age group (years) } \\
\text { 36-39 }\end{array}$ & 157 & 129,191 & 129 & 106,158 & 119 & 98,145 & 121 & 93,158 & 98 & 88,109 & 111 & 105,118 & $<0.0001$ \\
\hline $40-49$ & 148 & 131,169 & 117 & 98,139 & 128 & 116,141 & 100 & 79,127 & 108 & 101,115 & 107 & 103,111 & \\
\hline $50-59$ & 136 & 121,152 & 124 & 100,154 & 122 & 110,136 & 104 & 93,116 & 108 & 101,115 & 107 & 103,111 & \\
\hline 60-69 & 108 & 92,126 & 109 & 77,155 & 95 & 83,107 & & 67,95 & 80 & 73,88 & 87 & 83,92 & \\
\hline $70-79$ & & 67,116 & 77 & 52,114 & & 60,82 & & 55,81 & & 58,77 & & 59,69 & \\
\hline$P$ valuet & \multicolumn{2}{|c|}{$<0.0001$} & \multicolumn{2}{|c|}{$<0.0001$} & \multicolumn{2}{|c|}{$<0.0001$} & \multicolumn{2}{|c|}{$<0.0001$} & \multicolumn{2}{|c|}{$<0.0001$} & \multicolumn{2}{|c|}{$<0.0001$} & \\
\hline Women & \multirow{2}{*}{\multicolumn{2}{|c|}{$(n 516)$}} & \multicolumn{2}{|c|}{ (n 218) } & \multicolumn{2}{|c|}{$(n 793)$} & \multicolumn{2}{|c|}{ (n 329) } & \multicolumn{2}{|c|}{$(n$ 1405) } & \multicolumn{2}{|c|}{ (n 4457) } & \\
\hline Age group (y & & & & & & & & & & & & & \\
\hline 36-39 & 30 & 23,38 & 43 & 35,52 & 26 & 21,32 & & 15,38 & 26 & 23,30 & 28 & 26,30 & 0.0019 \\
\hline $40-49$ & 35 & 31,40 & 28 & 23,34 & 30 & 26,34 & 25 & 21,31 & 26 & 24,29 & 26 & 25,28 & \\
\hline $50-59$ & 61 & 53,70 & 52 & 42,65 & 49 & 44,55 & 51 & 43,60 & 51 & 47,55 & 50 & 48,52 & \\
\hline $60-69$ & 97 & 84,111 & 74 & 57,95 & 62 & 56,69 & 72 & 60,86 & 59 & 54,65 & 61 & 58,64 & \\
\hline 70-79 & 79 & 63,99 & & 24,65 & & 47,66 & 58 & 46,73 & 46 & 41,52 & 55 & 51,59 & \\
\hline$P$ valuet & \multicolumn{2}{|c|}{$<0.0001$} & \multicolumn{2}{|c|}{$<0.0001$} & \multicolumn{2}{|c|}{$<0.0001$} & $<0$ & 0001 & \multicolumn{2}{|c|}{$<0.0001$} & \multicolumn{2}{|c|}{$<0.0001$} & \\
\hline
\end{tabular}

s-Ferritin, serum ferritin.

${ }^{*}$ Test for differences between geographical areas adjusted for age and ethnicity.

tTest for differences between age groups.

Table 3 s-Ferritin by dietary pattern for 14630 participants in northern Norway: the SAMINOR survey

\begin{tabular}{|c|c|c|c|c|c|c|c|c|c|c|c|}
\hline & \multicolumn{11}{|c|}{$\mathrm{s}$-Ferritin $(\mu \mathrm{g} / \mathrm{l})$} \\
\hline & \multicolumn{2}{|c|}{ Reindeer } & \multicolumn{2}{|c|}{ Fish } & \multicolumn{2}{|c|}{ Average } & \multicolumn{2}{|c|}{ Fruit/vegetables } & \multicolumn{2}{|c|}{ Western/marine } & \multirow[b]{2}{*}{$P$ value* } \\
\hline & Mean & $95 \% \mathrm{Cl}$ & Mean & $95 \% \mathrm{Cl}$ & Mean & $95 \% \mathrm{Cl}$ & Mean & $95 \% \mathrm{Cl}$ & Mean & $95 \% \mathrm{Cl}$ & \\
\hline Men & \multicolumn{2}{|c|}{$(n$ 376) } & \multicolumn{2}{|c|}{$(n 705)$} & \multicolumn{2}{|c|}{$(n$ 1710) } & \multicolumn{2}{|c|}{$(n 700)$} & \multicolumn{2}{|c|}{ (n 2422) } & \\
\hline Age group (years) & & & & & & & & & & & \\
\hline $36-39$ & 169 & 140,203 & 126 & 98,160 & 108 & 97,121 & 117 & 100,137 & 114 & 105,123 & $<0.0001$ \\
\hline $40-49$ & 153 & 133,175 & 112 & 99,131 & 109 & 102,116 & 104 & 95,114 & 108 & 103,114 & \\
\hline $50-59$ & 146 & 129,164 & 113 & 102,125 & 109 & 103,117 & 109 & 99,119 & 107 & 102,112 & \\
\hline $60-69$ & 112 & 92,135 & 80 & 73,89 & 86 & 80,94 & 94 & 84,106 & 87 & 81,94 & \\
\hline $70-79$ & 84 & 64,108 & 76 & 66,89 & 66 & 59,73 & 68 & 56,83 & 68 & 61,76 & \\
\hline$P$ valuet & \multicolumn{2}{|c|}{$<0.0001$} & \multicolumn{2}{|c|}{$<0.0001$} & \multicolumn{2}{|c|}{$<0 \cdot 0001$} & \multicolumn{2}{|c|}{$<0.0001$} & \multicolumn{2}{|c|}{$<0.0001$} & \\
\hline Women & \multicolumn{2}{|c|}{$(n 370)$} & \multicolumn{2}{|c|}{$(n 980)$} & \multicolumn{2}{|c|}{$(n$ 1404) } & \multicolumn{2}{|c|}{$(n$ 1667) } & \multicolumn{2}{|c|}{ (n 1999) } & \\
\hline Age group (years) & & & & & & & & & & & \\
\hline $36-39$ & 32 & 24,44 & 30 & 23,40 & 30 & 26,34 & 28 & 25,31 & 25 & 23,28 & $<0.0001$ \\
\hline $40-49$ & 36 & 30,42 & 27 & 24,31 & 25 & 23,28 & 27 & 25,30 & 27 & 25,29 & \\
\hline $50-59$ & 61 & 51,72 & 53 & 48,57 & 50 & 46,54 & 47 & 44,51 & 51 & 48,54 & \\
\hline $60-69$ & 103 & 88,120 & 64 & 59,70 & 64 & 58,70 & 66 & 61,72 & 57 & 53,62 & \\
\hline $70-79$ & 77 & 56,104 & 55 & 48,61 & 54 & 48,62 & 60 & 52,66 & 50 & 45,57 & \\
\hline$P$ valuet & \multicolumn{2}{|c|}{$<0.0001$} & \multicolumn{2}{|c|}{$<0.0001$} & \multicolumn{2}{|c|}{$<0.0001$} & \multicolumn{2}{|c|}{$<0.0001$} & \multicolumn{2}{|c|}{$<0.0001$} & \\
\hline
\end{tabular}

s-Ferritin, serum ferritin.

${ }^{*}$ Test for differences between dietary patterns adjusted for age.

tTest for differences between age groups.

that in the other dietary clusters in both sexes. Transferrin saturation decreased significantly with increasing age, especially among men in the reindeer group.

\section{Fe levels influenced by dietary patterns}

Table 5 presents the results from multiple linear regression analyses for the effects of age, ethnic groups, geographical residence, BMI and dietary patterns on s-ferritin, stratified by sex. In this model, all independent variables were mutually adjusted for each of the other variables, men and women separately. For both sexes living inland, the reindeer dietary subgroup, overweight $\left(25 \cdot 0-29 \cdot 0 \mathrm{~kg} / \mathrm{m}^{2}\right)$ and obesity $\left(\geqslant 30 \cdot 0 \mathrm{~kg} / \mathrm{m}^{2}\right)$ were positively associated with s-ferritin. s-Ferritin among obese individuals was $25 \%$ higher in women $(\beta=0 \cdot 22)$ and $43 \%$ higher in men $(\beta=0 \cdot 31)$, compared with the normal weight group $\left(18 \cdot 5-25 \cdot 0 \mathrm{~kg} / \mathrm{m}^{2}\right)$. In women, the Sami I group was positively associated with higher s-ferritin.

In a final model, all independent variables were mutually adjusted for each other; in addition, an interaction 
Table 4 Transferrin saturation by dietary pattern for 14630 participants in northern Norway: the SAMINOR survey

\begin{tabular}{|c|c|c|c|c|c|c|c|c|c|c|c|}
\hline & \multicolumn{10}{|c|}{ Transferrin saturation (\%) } & \multirow[b]{3}{*}{$P$ value } \\
\hline & \multicolumn{2}{|c|}{ Reindeer } & \multicolumn{2}{|c|}{ Fish } & \multicolumn{2}{|c|}{ Average } & \multicolumn{2}{|c|}{ Fruit/vegetables } & \multicolumn{2}{|c|}{ Western/marine } & \\
\hline & Mean & $95 \% \mathrm{Cl}$ & Mean & $95 \% \mathrm{Cl}$ & Mean & $95 \% \mathrm{Cl}$ & Mean & $95 \% \mathrm{Cl}$ & Mean & $95 \% \mathrm{Cl}$ & \\
\hline Men & \multicolumn{2}{|c|}{$(n 376)$} & \multicolumn{2}{|c|}{$(n 705)$} & \multicolumn{2}{|c|}{$(n 1710)$} & \multicolumn{2}{|c|}{$(n 700)$} & \multirow{2}{*}{\multicolumn{2}{|c|}{ (n 2422) }} & \\
\hline Age group (years) & & & & & & & & & & & \\
\hline & $\begin{array}{l}31 \\
31\end{array}$ & $\begin{array}{l}27,34 \\
29,33\end{array}$ & $\begin{array}{l}27 \\
29\end{array}$ & 22,33 & $\begin{array}{l}29 \\
29\end{array}$ & $\begin{array}{l}27,31 \\
28,30\end{array}$ & 31 & $\begin{array}{l}27,34 \\
28,31\end{array}$ & $\begin{array}{l}28 \\
29\end{array}$ & $\begin{array}{l}27,29 \\
28,30\end{array}$ & $<0.04$ \\
\hline $\begin{array}{l}40-49 \\
50-59\end{array}$ & $\begin{array}{l}31 \\
29\end{array}$ & $\begin{array}{l}29,33 \\
28,31\end{array}$ & $\begin{array}{l}29 \\
31\end{array}$ & $\begin{array}{l}27,30 \\
29,32\end{array}$ & $\begin{array}{l}29 \\
30\end{array}$ & $\begin{array}{l}28,30 \\
29,31\end{array}$ & $\begin{array}{l}30 \\
30\end{array}$ & $\begin{array}{l}28,31 \\
28,31\end{array}$ & $\begin{array}{l}29 \\
29\end{array}$ & $\begin{array}{l}28,30 \\
29,30\end{array}$ & \\
\hline $60-69$ & 31 & 28,34 & 29 & 28,30 & 31 & 30,32 & 30 & 29,32 & 29 & 28,30 & \\
\hline $70-79$ & 27 & 23,30 & 29 & 27,30 & 29 & 27,30 & 30 & 28,33 & 29 & 28,31 & \\
\hline$P$ valuet & \multicolumn{2}{|c|}{$<0.0009$} & \multicolumn{2}{|c|}{$<0.08$} & \multicolumn{2}{|c|}{$<0.02$} & \multicolumn{2}{|c|}{$<0.04$} & \multicolumn{2}{|c|}{$<0.36$} & \\
\hline Women & \multirow{2}{*}{\multicolumn{2}{|c|}{$(n 370)$}} & \multirow{2}{*}{\multicolumn{2}{|c|}{$(n 980)$}} & \multicolumn{2}{|c|}{ (n 1404) } & \multirow{2}{*}{\multicolumn{2}{|c|}{$(n$ 1667) }} & \multirow{2}{*}{\multicolumn{2}{|c|}{ (n 1999) }} & \\
\hline Age group (years) & & & & & & & & & & & \\
\hline $36-39$ & 28 & 24,32 & 26 & 21,32 & 27 & 25,29 & 24 & 23,26 & 26 & 24,27 & $<0.02$ \\
\hline $40-49$ & 26 & 24,28 & 26 & 24,28 & 27 & 26,28 & 26 & 25,27 & 26 & 25,27 & \\
\hline $50-59$ & 26 & 25,28 & 27 & 26,28 & 28 & 27,29 & 27 & 26,28 & 26 & 26,27 & \\
\hline $60-69$ & 28 & 25,32 & 27 & 26,28 & 28 & 27,29 & 27 & 26,28 & 27 & 26,28 & \\
\hline $70-79$ & 24 & 21,26 & 28 & 27,30 & 26 & 25,28 & 27 & 25,29 & 27 & 25,28 & \\
\hline$P$ valuet & \multicolumn{2}{|c|}{$<0.34$} & \multicolumn{2}{|c|}{$<0.57$} & \multicolumn{2}{|c|}{$<0.12$} & \multicolumn{2}{|c|}{$<0.09$} & \multicolumn{2}{|c|}{$<0.67$} & \\
\hline
\end{tabular}

*Test for differences between dietary patterns adjusted for age.

tTest for differences between age groups.

Table 5 Effect of age, ethnicity, geographical area, BMI and dietary pattern on serum ferritin level: results from multiple linear regression analyses

\begin{tabular}{|c|c|c|c|c|c|c|}
\hline & \multicolumn{3}{|c|}{ Women } & \multicolumn{3}{|c|}{ Men } \\
\hline & $\beta$ Coefficient $^{*}$ & $95 \% \mathrm{Cl}$ & $P$ value & $\beta$ Coefficient $^{*}$ & $95 \% \mathrm{Cl}$ & $P$ value \\
\hline \multicolumn{7}{|l|}{ Age group (years) } \\
\hline $36-39$ & Ref. & & & Ref. & & \\
\hline $40-49$ & -0.02 & $-0.09,0.05$ & 0.51 & -0.01 & $-0.08,0.06$ & 0.75 \\
\hline $50-59$ & 0.58 & $0.51,0.65$ & $<0.0001$ & -0.03 & $-0.09,0.04$ & 0.44 \\
\hline $60-69$ & 0.79 & $0.71,0.86$ & $<0.0001$ & -0.25 & $-0.32,-0.19$ & $<0.0001$ \\
\hline $70-79$ & 0.62 & $0.54,0.70$ & $<0.0001$ & $-0 . \overline{51}$ & $-0.59,-0.44$ & $<0.0001$ \\
\hline \multicolumn{7}{|l|}{ Ethnic group } \\
\hline Non-Sami & Ref. & & & Ref. & & \\
\hline Sami I & 0.08 & $0.009,0.15$ & 0.03 & 0.006 & $-0.06,0.06$ & 0.98 \\
\hline Sami II & -0.03 & $-0.08,0.01$ & $0 \cdot 18$ & -0.028 & $-0.07,0.01$ & 0.17 \\
\hline \multicolumn{7}{|l|}{ Geographical area } \\
\hline Coastal & Ref. & & & Ref. & & \\
\hline Inland & 0.05 & $0.002,0.11$ & 0.03 & 0.13 & $0.08,0.17$ & $<0.0001$ \\
\hline \multicolumn{7}{|l|}{ BMI $\left(\mathrm{kg} / \mathrm{m}^{2}\right)$} \\
\hline $18 \cdot 5-25 \cdot 0$ & Ref. & & & Ref. & & \\
\hline$<18.5$ & 0.03 & $-0.18,0.24$ & 0.78 & 0.04 & $-0.24,0.31$ & 0.80 \\
\hline $25 \cdot 0-29 \cdot 0$ & 0.07 & $0.03,0.12$ & 0.0005 & $0 \cdot 16$ & $0.12,0.20$ & $<0.0001$ \\
\hline$\geq 30 \cdot 0$ & 0.22 & $0.17,0.27$ & $<0.0001$ & 0.31 & $0.26,0.35$ & $<0.0001$ \\
\hline \multicolumn{7}{|l|}{ Dietary pattern } \\
\hline Average & Ref. & & & Ref. & & \\
\hline Reindeer & 0.17 & $0.06,0.27$ & 0.002 & 0.22 & $0.14,0.31$ & $<0.0001$ \\
\hline Fish & 0.03 & $-0.04,0.09$ & 0.41 & 0.02 & $-0.04,0.08$ & 0.51 \\
\hline Fruit/vegetables & 0.002 & $-0.05,0.05$ & 0.95 & 0.013 & $-0.05,0.07$ & 0.67 \\
\hline Western/marine & -0.02 & $-0.07,0.03$ & 0.49 & 0.008 & $-0.03,0.05$ & 0.70 \\
\hline
\end{tabular}

Ref., reference group.

*Mutually adjusted for each of the other variables, men and women separately.

term was included between the effects of ethnicity and geographical residence (Table 6). In this model, the positive association between s-ferritin and Sami I disappeared. Inland residence was, however, significant among men. Other lifestyle factors such as light/hard physical activity, and, in addition, for women the number of childbirths, were considered as confounders. However, they were not added to the model as they did not change the associations presented in Tables 5 and 6.

\section{Discussion}

In the present cross-sectional survey of the Sami and non-Sami population in northern Norway, we have observed an association between s-ferritin level and several lifestyle factors. Participants who frequently consumed reindeer meat and other reindeer products had higher levels of s-ferritin than did individuals with other dietary patterns. However, the reindeer pattern individuals 
Table 6 Effect of age, ethnicity where interaction of geographical area is included, BMI and dietary pattern on serum ferritin level: results from multiple linear regression analyses

\begin{tabular}{|c|c|c|c|c|c|c|}
\hline & \multicolumn{3}{|c|}{ Women } & \multicolumn{3}{|c|}{ Men } \\
\hline & $\beta$ Coefficient $^{*}$ & $95 \% \mathrm{Cl}$ & $P$ value & $\beta$ Coefficient $^{*}$ & $95 \% \mathrm{Cl}$ & $P$ value \\
\hline \multicolumn{7}{|l|}{ Age group (years) } \\
\hline $36-39$ & Ref. & & & Ref. & & \\
\hline $40-49$ & -0.02 & $-0.09,0.05$ & 0.52 & -0.01 & $-0.07,0.06$ & $0 \cdot 74$ \\
\hline $50-59$ & 0.58 & $0.51,0.65$ & $<0.0001$ & -0.02 & $-0.09,0.04$ & 0.45 \\
\hline $60-69$ & 0.79 & $0.72,0.87$ & $<0.0001$ & -0.25 & $-0.32,-0.19$ & $<0.0001$ \\
\hline $70-79$ & 0.63 & $0.55,0.71$ & $<0.0001$ & -0.51 & $-0.59,-0.44$ & $<0.0001$ \\
\hline \multicolumn{7}{|l|}{ Ethnic group } \\
\hline Non-Sami & Ref. & & & Ref. & & \\
\hline Sami I & 0.01 & $-0 \cdot 08,0 \cdot 10$ & $0 \cdot 83$ & -0.05 & $-0.12,0.03$ & 0.25 \\
\hline Sami II & -0.04 & $-0.089,0.01$ & $0 \cdot 10$ & -0.02 & $-0.07,0.02$ & 0.28 \\
\hline \multicolumn{7}{|l|}{ Geographical area } \\
\hline Coastal & Ref. & & & Ref. & & \\
\hline Inland & 0.01 & $-0.05,0.08$ & 0.72 & $0 \cdot 11$ & $0 \cdot 06,0 \cdot 17$ & $<0.0001$ \\
\hline \multicolumn{7}{|l|}{ Interaction term } \\
\hline Sami I × inland & $0 \cdot 16$ & $0.03,0.30$ & 0.02 & $0 \cdot 10$ & $-0.02,0.22$ & 0.09 \\
\hline Sami II $\times$ inland & 0.08 & $-0.05,0.22$ & $0 \cdot 23$ & -0.03 & $-0.15,0.09$ & $0 \cdot 64$ \\
\hline \multicolumn{7}{|l|}{ BMI $\left(\mathrm{kg} / \mathrm{m}^{2}\right)$} \\
\hline $18 \cdot 5-25 \cdot 0$ & Ref. & & & Ref. & & \\
\hline$<18.5$ & 0.03 & $-0 \cdot 18,0 \cdot 24$ & $0 \cdot 80$ & 0.04 & $-0.24,0.31$ & 0.79 \\
\hline $25 \cdot 0-29 \cdot 0$ & 0.07 & $0 \cdot 03,0.12$ & 0.0004 & $0 \cdot 16$ & $0 \cdot 12,0 \cdot 20$ & $<0.0001$ \\
\hline$\geq 30 \cdot 0$ & $0 \cdot 22$ & $0 \cdot 17,0.27$ & $<0.0001$ & $0 \cdot 31$ & $0.26,0.35$ & $<0.0001$ \\
\hline \multicolumn{7}{|l|}{ Dietary pattern } \\
\hline Average & Ref. & & & Ref. & & \\
\hline Reindeer & $0 \cdot 13$ & $0.02,0.24$ & 0.02 & $0 \cdot 221$ & $0 \cdot 11,0 \cdot 30$ & $<0.0001$ \\
\hline Fish & 0.03 & $-0.04,0.09$ & 0.42 & 0.02 & $-0.04,0.08$ & 0.50 \\
\hline Fruit/vegetables & 0.002 & $-0.05,0.05$ & 0.93 & 0.013 & $-0.04,0.07$ & 0.63 \\
\hline Western/marine & -0.02 & $-0.07,0.03$ & 0.48 & 0.008 & $-0.03,0.05$ & 0.69 \\
\hline
\end{tabular}

Ref., reference group.

*Mutually adjusted for each of the other variables, men and women separately.

also had the highest proportion of subjects with overweight and obesity.

In the SAMINOR survey, food consumption frequency was estimated, although the questionnaire did not cover the total diet. The questionnaire was not designed to cover the total diet but rather to gather information on the use of selected foods relevant for the northern area. The analyses of food patterns, rather than of single nutrients, are considered an alternative way to focus on diet and health outcomes, because nutrient intake could not be derived from the $\mathrm{FFQ}^{(12,13)}$. Brustad et al. ${ }^{(12)}$ have published dietary pattern analyses from the SAMINOR survey, which showed that ethnicity did not play a major role in predicting dietary patterns, except for the reindeer group of foods in inland areas ${ }^{(11,14)}$. For coastal settlements, marine fish and different types of meat constitute primary nutritional sources for both ethnic groups. Dietary traditions are important markers for cultural identity. Because of changes in occupation among indigenous people, such as from primary trades to service professions, traditions are being altered that can affect dietary patterns. In addition, increased access to many different food products also establishes new food habits. These changes are global and are described, among others, by Kuhnlein et $a l^{(15)}$ for Arctic indigenous people in Canada. However, in inland areas in northern Norway, reindeer meat is easily accessible and has a high Fe content of $3.8 \mathrm{mg} /$
$100 \mathrm{~g}$ wet weight ${ }^{(16)}$. Frequent use affects Fe levels. The present study showed that geographical residence, inland, had a significant influence in both sexes. Sami I women had significantly higher s-ferritin levels compared with the comparable groups. To examine interactions between ethnic groups and those living inland, interaction terms were created. The combined effect of both exposures is less than the combination of their separate effects, which means that it is unlikely that ethnicity is the explanation for higher s-ferritin levels. The causality is most probably explained by dietary habits, geographical settlement and BMI. It is therefore unlikely that genetic inheritance will explain the observed differences between ethnic groups.

Overweight and obesity had a significant effect on s-ferritin in both sexes. The study showed a high prevalence of overweight and obesity in the study population. The reindeer pattern had the highest proportion of subjects with overweight. A newly published article from the same series, the SAMINOR study, showed that both obesity and central obesity were most pronounced in Sami women, whereas the opposite was true for men ${ }^{(17)}$. General obesity among Sami women has been previously reported by Njølstad et al. ${ }^{(18)}$ in 1998. However, data on the prevalence of metabolic syndrome in this population are not available at present, but can be a crucial confounding factor in this context. The metabolic syndrome 
is a cluster of unfavourable health factors such as obesity, insulin resistance, dyslipidaemia and hypertension ${ }^{(19,20)}$. These core components are all metabolically related cardiovascular risk factors. Individuals with metabolic syndrome have an increased risk of developing atherosclerosis and CVD. s-Apoferritin is a part of measured s-ferritin and is an acute-phase reactant protein elevated in infectious, inflammatory and malignant diseases. High levels of acute-phase reactants such as high-sensitivity CRP and Fe stores have been reported to correlate with metabolic syndrome ${ }^{(21,22)}$. The presumed reason is that inflammation plays a key role in the development of atherosclerosis. Obesity-related insulin resistance and inflammation are generally considered to be central elements in the pathogenesis of metabolic syndrome ${ }^{(23,24)}$. Elevated s-ferritin levels are also associated with increased diabetes mellitus risk among healthy individuals, where $\mathrm{s}$-ferritin is an important and independent predictor of the development of diabetes ${ }^{(25)}$.

Fe may contribute to the pathogenesis of type 2 diabetes mellitus by inducing oxidative stress and interfering with insulin secretion. Glucose and macronutrient intakes cause oxidative stress and inflammatory changes ${ }^{(23)}$. The increased concentrations of TNF- $\alpha$ and IL-6, associated with obesity and type 2 diabetes, might interfere with insulin action by suppressing insulin signal transduction. This might interfere with the anti-inflammatory effect of insulin, which in turn might promote inflammation ${ }^{(23)}$. Chronic overnutrition (obesity) might thus be a proinflammatory state with oxidative stress.

The pro-inflammatory state of obesity and metabolic syndrome originates with excessive energy intake and is probably a result of overnutrition leading to clinical and biochemical manifestations of the metabolic syndrome.

However, the study revealed a few participants with elevated CRP. Nevertheless, the reindeer pattern individuals continued to have significantly higher s-ferritin after including the interaction term as well. This was the case in both sexes, although overweight and obesity were not as pronounced among Sami men. Another factor that affects Fe levels is coffee consumption. A high rate of intake inhibits $\mathrm{Fe}$ absorption ${ }^{(8,26)}$. The habit of coffee consumption is prevalent among the Sami I group and reindeer pattern members, but it has no apparent negative association with s-ferritin. On the other hand, Nystad et al. ${ }^{(27)}$ found that total coffee consumption in the same population was positively associated with total cholesterol levels. Dyslipidaemia may therefore be a confounding variable in this relationship.

Food patterns and health-related behaviours such as the level of physical activity may confound an association with s-ferritin. In a 1993-1994 Norwegian survey of the adult population, a quantitative FFQ was used to evaluate dietary habits and to relate these to other lifestyle factors ${ }^{(28)}$. That survey showed that daily total intakes of vitamins $\mathrm{B}_{1}$ and $\mathrm{B}_{2}$, $\mathrm{Ca}$ and $\mathrm{Fe}$ by men and women were higher among the youngest age groups compared with the older participants. In women, intakes of vitamin $\mathrm{C}$ was highest in the oldest age groups. However, regardless of age, women did not have adequate intakes of vitamin $\mathrm{D}$ and $\mathrm{Fe}$. In another study conducted in 1996 of breast cancer among Norwegian female citizens, dietary habits were analysed on the basis of an FFQ involving approximately 10000 women $^{(29)}$. In addition, the study showed that older women did have a more vitamin C-rich diet than the younger participants. Although these surveys were national, they did not focus specifically on ethnic groups or geographical areas. A smaller study conducted among a Sami population living in traditional reindeer herding areas of northern Norway concluded that, traditionally, there is low consumption of fruit and vegetables among inhabitants of northern Norway, especially among the elderly ${ }^{(30)}$. However, the sample size was small.

\section{Conclusion}

The present study shows that there is an association between s-ferritin and lifestyle factors. High level of s-ferritin may reflect high intake of reindeer meat. However, no causal relationships between $\mathrm{Fe}$ stores and reindeer meat consumption can be inferred from the present study. The differences in Fe status described earlier between inland Sami and non-Sami can be explained by several factors such as food habits, age and obesity. Clearly, being overweight and obese are associated with s-ferritin levels. On the basis of our findings, additional analyses on metabolic syndrome and $\mathrm{Fe}$ overload in the present population should be carried out.

\section{Acknowledgements}

Funding for this project was provided by the Norwegian Ministry of Health. The authors have no conflict of interest to declare. E.L. and A.R.B. designed and conducted the research; A.R.B. and M.M. analysed the data; A.R.B., M.B., M.M. and E.L. wrote the paper; A.R.B. had primary responsibility for the final content. All authors read and approved the final manuscript. The authors are indebted to the participants in the SAMINOR study who made this research possible. They also thank the staff at the Department of Clinical Chemistry, University Hospital of Northern Norway, for technical assistance and careful evaluation of the blood tests.

\section{References}

1. Milman N, Byg KE, Mulvad G et al. (2001) Iron status markers in 224 indigenous Greenlanders: influence of age, residence and traditional foods. Eur J Haematol 66, $115-125$. 
2. Broderstad AR Melhus M \& Lund E (2007) Iron status in a multiethnic population (age 36-80 years) in northern Norway: the SAMINOR study. Eur J Haematol 79, 447-454.

3. Milman N, Byg KE \& Ovesen L (2000) Iron status in Danes 1994. II: Prevalence of iron deficiency and iron overload in 1319 Danish women aged 40-70 years. Ann Hematol 79, 612-621.

4. Whitfield JB, Treloar S, Zhu G et al. (2003) Relative importance of female-specific and non-female-specific effects on variation in iron stores between women. $\mathrm{Br} J$ Haematol 120, 860-866.

5. Milman N (1996) Serum ferritin in Danes: studies of iron status from infancy to old age, during blood donation and pregnancy. Int J Hematol 63, 103-135.

6. Aubert V (1978). Den samiske befolkning i Nord-Norge/ The Lappish population in Northern Norway. http://www. ssb.no/histstat/art_107.pdf (accessed September 2007).

7. Lund E, Melhus M, Hansen KL et al. (2007) Population based study of health and living conditions in areas with both Sami and Norwegian populations - The SAMINOR Study. Int J Circumpolar Health 66, 89-184.

8. Milman N, Ovesen L, Byg KE et al. (1999) Iron status in Danes updated 1994. I: Prevalence of iron deficiency and iron overload in 1332 men aged $40-70$ years. Ann Hematol 78, 393-400.

9. World Health Organization/UNICEF/United Nations University (2001) Iron Deficiency Anaemia Assessment, Prevention, and Control. A Guide for Programme Managers (WHO/ NHD/01.3). Geneva: WHO.

10. World Health Organization (2000) Obesity: Prevention and Managing the Global Epidemic. Report of a WHO Consultation. WHO Technical Report Series no. 894. Geneva: WHO.

11. Brustad M, Parr DL, Melhus M et al. (2008) Dietary patterns in the population living in the Sámi core areas of Norway the SAMINOR study. Int J Circumpolar Health 67, 1-98.

12. Newby PK \& Tucker KL (2004) Empirically derived eating patterns using factor or cluster analysis: a review. Nutr Rev 62, 177-203.

13. Jacques PF \& Tucker KL (2001) Are dietary patterns useful for understanding the role of diet in chronic disease? $\mathrm{Am} \mathrm{J}$ Clin Nutr 73, 1-2.

14. Brustad M, Parr CL, Melhus M et al. (2007) Childhood diet in relation to Sámi and Norwegian ethnicity in northern and midNorway - the SAMINOR study. Public Health Nutr 5, 1-8.

15. Kuhnlein HV, Receveur O, Soueida R et al. (2004) Artic indigenous peoples experience the nutrition transition with changing dietary patterns and obesity. J Nutr 134, $1447-1453$.

16. The Norwegian Food Safety Authority, The Norwegian Directorate of Health \& University of Oslo (2006) The
Norwegian Food Composition Table 2006. http://matportalen. no/matvaretabellen/index_html/main_view_eng

17. Nystad T, Melhus M, Brustad M et al. (2010) Ethnic differences in the prevalence of general and central obesity among the Sami and Norwegian populations: the SAMINOR study. Scand J Public Health 38, 17-24.

18. Njølstad I, Arnesen E \& Lund-Larsen PG (1998) Cardiovascular diseases and diabetes mellitus in different ethnic groups: the Finnmark study. Epidemiology 9, 550-556.

19. Alberti KGM, Zimmet P \& Shaw J (2006) Metabolic syndrome - a new world-wide definition. A consensus Statement from the International Diabetes federation. Diabet Med 23, 469-480.

20. Wilsgaard T \& Jacobsen BK (2007) Lifestyle factors and incident metabolic syndrome. The Troms $\varnothing$ Study. Diabetes Res Clin Pract 78, 217-224.

21. Mainous AG, Wells BJ, Everett CJ et al. (2004) Association of ferritin and lipids with C-reactive protein. Am J Cardiol 93, 559-562.

22. González AS, Guerrero BD, Soto BM et al. (2006) Metabolic syndrome, insulin resistance and the inflammation markers C-reactive protein and ferritin. Eur J Clin Nutr 60, 802-809.

23. Dandona P, Aljada A \& Bandyopadhyay A (2004) Inflammation: the link between insulin resistance, obesity and diabetes. Trends Immunol 25, 4-7.

24. Mainous AG 3rd, Wells BJ, Everett CJ et al. (2004) Association of ferritin and lipids with C-reactive protein. Am J Cardiol 93, 559-562.

25. Forouhi NG, Harding AH, Allison M et al. (2007) Elevated serum ferritin levels predict new-onset type 2 diabetes: results from the EPIC-Norfolk prospective study. Diabetologia 50, 949-956.

26. Skikne B \& Baynes RD (1994) Iron absorption. In Iron Metabolism in Health and Disease, pp. 151-187 [JH Brock, JW Halliday, MJ Pippard et al., editors]. London: Saunders.

27. Nystad T, Melhus M, Brustad M et al. (2010) The effect of coffee consumption on serum total cholesterol in the Sami and Norwegian populations. Public Health Nutr 13, $1818-1825$.

28. Johansson L, Solvoll K, Bjørneboe G-E et al. (1997) Dietary habits among Norwegian men and women. Scand J Nutr 41, 63-70.

29. Hjartåker A \& Lund E (1998) Relationship between dietary habits, age, lifestyle and socio-economic status among adult Norwegian women. The Norwegian Women and Cancer Study. Eur J Clin Nutr 52, 565-572.

30. Nilsen H, Utsi E \& Bonaa KH (1999) Dietary and nutrient of a Sámi population living in traditional reindeer herding areas in north Norway. Comparisons with a group of Norwegians. Int J Circumpolar Health 58, 120-133. 